\title{
Evaluating of the predictors of high blood pressure in children and adolescence: Findings from Iranian health care system reform plan
}

\section{Shahnaz Taghizadeh}

Tabriz University of Medical Sciences Faculty of Health and Nutrition

Mahdieh Abbasalizad Farhangi ( $\nabla$ abbasalizad_m@yahoo.com )

Tabriz University of Medical Sciences https://orcid.org/0000-0002-7036-6900

\section{Fathollah Pourali}

Azarbaijan Shahid Madani University

mohammad asghari jafarabadi

Tabriz University of Medical Sciences

\section{Research note}

Keywords: hypertension, children, adolescents, Iranian health care reform system

Posted Date: July 16th, 2020

DOI: https://doi.org/10.21203/rs.3.rs-42619/v1

License: (c) (i) This work is licensed under a Creative Commons Attribution 4.0 International License. Read Full License 


\section{Abstract}

Objective: The aim of this study is the evaluate of dietary pattern and life style associated factors in predicting hypertension among overweight and obese pediatrics. In the current cross-sectional study, 425 overweight and obese children and adolescents aged 6 to 18 years were enrolled. The predictors of blood pressure were included in the scoring algorithm.

Results: The results showed that SBP and DBP had directly correlation with age $(p<0.001, p<0.001)$ and BMI $(p<0.005, p<0.007)$ respectively. Moreover, DBP was in correlation with fruit consumption of less than 2 serving per day versus never consumption $(p=0.014, B=0.444)$, fruit consumption of more than 2 serving per day versus never consumption $(p=0.014, B=0.480)$, and vegetable consumption less than 3 serving per day versus never consumption ( $p=0.045, B=-0.374)$. Also, results showed that DBP had significant correlation with fast foods $/ \mathrm{junk}$ foods consumption of 1-2 items per week versus almost every day consumption $(p=0.047, B=-0.177)$. This final model could predict $32.1 \%$ of hypertension by SBP and DBP (R2 =0.321). According to our findings, consumption of lower vegetables and fruits, higher amounts of fast foods, higher age and BMI could be potent predictors of high blood pressure among Iranian children and adolescents.

\section{Introduction}

Blood pressure (BP) is one of the most important problems affecting obese children and adolescents. The prevalence of hypertension (HTN) in obese children ranges from $19-22 \%$, compared to $4-6 \%$ in normal weight children [1, 2]. However, the prevalence of HTN among Iranian children and adolescents was estimated to be $8.9 \%$ in overall [3]. Giving that, currently, Iran is undergoing a nutritional transition [4] and nutritional transition in developing countries has led to overweight and obesity problems [5], therefore, it seems that Iranian children are at higher risk of developing cardiovascular disease risk factors where the obesity-related HTN may play a significant role. Studies show that HTN is significantly associated with the combination of genetic, environment, behavioral and dietary factors [6]. Menghetti et al, demonstrated that obese children and adolescents had higher risk of developing HTN apparently four times greater than normal weight subjects. They also demonstrated that fruit and salad consumption has a protective effect against hypertension [7]. Moore et al demonstrated that consuming more than two servings of dairy and more than three servings of fruits and vegetables daily is associated with lower BP in childhood and a $36 \%$ lower risk of developing high blood pressure (HBP) by young adulthood [8].

Since the 1970s, the National Heart, Lung, and Blood Institute has recommended measurement of BP in healthy children as part of routine health maintenance, Programs that combine diet and physical activity (PA) can have a beneficial effect on SBP, as is shown in several studies designed to prevent childhood obesity and address cardiometabolic risk factors [9].

In the current study, we hypothesized that SBP and DBP both increase with sedentary lifestyle behaviors, increased age, increased consumption fast foods and reduced consumption of fruit, vegetable, dairy products and frequent meals in childhood and teens. However, developing a model which considers these factors in a combined model not in isolate form, could best predict a usual life style module. We assessed the correlation between known nutritional and lifestyle associated risk factors of BP in a life-style scoring algorithm among children and adolescents.

\section{Methods}


The present study is a descriptive cross-sectional study that was performed in 2018, using the Sib system (https://sib.tbzmed.ac.ir/home/) related to the health care reform plan investigating the anthropometric information, demographic factors, dietary intakes and lifestyle associated factors in 425 overweight and obese children and adolescents (254 males and 179 females), 6 to 18 years old, referred to Shahid-Bakeri Health center, a large health care system representative of the population of four distinct with different socioeconomic status, in Tabriz that selected by using convenience sampling method. The exclusion criteria were renal or liver disease, diabetes, heart disease, thyroid dysfunctions, pregnancy and smoking and taking steroid medications. The parental and adolescents consent obtained for this study. Demographic information and detailed diet history were recorded and weight, height and blood pressure were measured by expert dietitian.

dietary intake, PA, and lifestyle behaviors were assessed according the health care system reform plan. HBP was defined according to the fourth report of the diagnosis and treatment of hypertension in American children and adolescents by gender-specific BP tables[10]. Accordingly, SBP and DBP $<90, \geq 90$ and $<95$, and $\geq 95$ percentile considered as normal, prehypertension and hypertension respectively. Also, overweight and obesity was defined as having $+1 \mathrm{Z}$ score $<\mathrm{BMI}<+2 \mathrm{Z}$-score and $\mathrm{BMI}>+2 \mathrm{Z}$ score respectively. The questionnaire, that use in the Iranian health care system reform plan, approved by the Ministry of Health and Medical Education of Iran [11] and contained 7 questions and the first, second and third option of each question has 0,1 and 2 scores respectively. The details of questions are provided in Table S1. The total scores of all questions are summed up and the life style algorithm final score ranges between 0 to14.

\section{Statistical Analysis}

The data were expressed as mean (SE) and frequency (\%) for quantitative and qualitative variables, respectively (nominal or ordinal). Normality of data was confirmed based on Kolmogorov-Smirnov. Data analysis was performed using statistical software STATA (MP 4.2 potable 2017). Using the univariate and Hierarchical linear multivariable regression models, the effect of SBP and DBP predictors was studied separately and adjusted with other variables, respectively. We classified the variables into groups according to the Hierarchical Multivariable Linear Regression Model and examined the factors based on the three models.

\section{Result}

The characteristics of the participants are presented in Table S2. The mean ( \pm SD) age of 10.62$) \pm 3.45), 86.12 \%$ of them had normal BP, $9.41 \%$ prehypertension and $4.47 \%$ hypertension. In general, $19.88 \%$ of them had prehypertension and hypertension. Table 1 shows that elevated SBP and DBP were associated with increased consumption of fruits, vegetables; while reduced SBP and DBP was in parallel of reduced consumption of fast foods. Accordingly, reduced SBP were associated with reduced TV/PC watching. In the girls, reduced SBP and DBP values was associated with reduced consumption of fast foods, TV/PC watching and increased PA. Moreover, reduced SBP was associated with increased vegetables consumption. Table 2 shows the correlation between SBP and DBP with underlying variables, lifestyle and dietary. The results show that, SBP and DBP had positive correlation with BMI and PA and inverse correlation with watching TV/PC. Also, DBP, had positive correlation with consumption of fruit. Given that, the effect of any variable can be influenced by one or a group of variables, so we used the Hierarchical regression model in the final step. 
Table 1

The mean systolic and diastolic blood pressure in participants; according to lifestyle

\begin{tabular}{|c|c|c|c|c|c|c|}
\hline \multirow[t]{2}{*}{$\begin{array}{l}\text { Lifestyle (food intake and physical } \\
\text { activity) }\end{array}$} & \multicolumn{3}{|c|}{$\begin{array}{l}\text { Boys } \\
(n=254)\end{array}$} & \multicolumn{3}{|c|}{$\begin{array}{l}\text { Girls } \\
(n=179)\end{array}$} \\
\hline & $n$ & SBP & DBP & $\mathbf{n}$ & SBP & DBP \\
\hline & & Mean \pm SE & Mean \pm SE & & Mean \pm SE & Mean \pm SE \\
\hline \multicolumn{7}{|l|}{ Fruit } \\
\hline Rarely/never & 3 & $86.66 \pm 5.77$ & $\begin{array}{l}46.66 \pm \\
5.77\end{array}$ & 2 & $100 \pm 0$ & $60 \pm 0$ \\
\hline$<2$ serving / day & 38 & $\begin{array}{l}97.36 \pm \\
13.54\end{array}$ & $60 \pm 10.06$ & 32 & $\begin{array}{l}101.56 \pm \\
11.46\end{array}$ & $\begin{array}{l}63.87 \pm \\
10.31\end{array}$ \\
\hline$\geq 2$ serving / day & 205 & $\begin{array}{l}100.78 \pm \\
14.35\end{array}$ & $\begin{array}{l}63.12 \pm \\
9.65\end{array}$ & 145 & $\begin{array}{l}99.77 \pm \\
10.88\end{array}$ & $\begin{array}{l}61.79 \pm \\
8.47\end{array}$ \\
\hline \multicolumn{7}{|l|}{ Vegetable } \\
\hline Rarely/never & 4 & $97.5 \pm 17.07$ & $60.25 \pm 15$ & 4 & $\begin{array}{l}103.75 \pm \\
7.5\end{array}$ & $62.5 \pm 5$ \\
\hline$<3$ serving / day & 66 & $\begin{array}{l}97.57 \pm \\
15.22\end{array}$ & $\begin{array}{l}60.30 \pm \\
1.14\end{array}$ & 60 & $\begin{array}{l}103.41 \pm \\
12.02\end{array}$ & $64.4 \pm 9.40$ \\
\hline$\geq 3$ serving / day & 176 & $\begin{array}{l}101.19 \pm \\
13.78\end{array}$ & $\begin{array}{l}63.23 \pm \\
9.59\end{array}$ & 115 & $98.23 \pm 10$ & $\begin{array}{l}60.95 \pm \\
8.37\end{array}$ \\
\hline \multicolumn{7}{|l|}{ Dairy product } \\
\hline Rarely/never & 3 & $90 \pm 0$ & $\begin{array}{l}56.66 \pm \\
5.77\end{array}$ & 3 & $\begin{array}{l}103.33 \pm \\
5.77\end{array}$ & $\begin{array}{l}63.33 \pm \\
5.77\end{array}$ \\
\hline$<3$ serving / day & 49 & $\begin{array}{l}103.16 \pm \\
13.52\end{array}$ & $64.38 \pm 9.1$ & 55 & $\begin{array}{l}102.38 \pm \\
11.02\end{array}$ & $\begin{array}{l}64.07 \pm \\
8.29\end{array}$ \\
\hline$\geq 3$ serving / day & 194 & $\begin{array}{l}99.56 \pm \\
14.43\end{array}$ & $\begin{array}{l}62.03 \pm \\
10.05\end{array}$ & 121 & $\begin{array}{l}99.06 \pm \\
10.79\end{array}$ & $\begin{array}{l}61.32 \pm \\
8.97\end{array}$ \\
\hline \multicolumn{7}{|l|}{ Fast foods /junk foods } \\
\hline almost every day & 16 & $\begin{array}{l}101.25 \pm \\
14.88\end{array}$ & $\begin{array}{l}60.53 \pm \\
11.57\end{array}$ & 21 & $\begin{array}{l}102.38 \pm \\
11.02\end{array}$ & $\begin{array}{l}64.28 \pm \\
11.64\end{array}$ \\
\hline $1-2$ items/ week & 67 & $\begin{array}{l}100.52 \pm \\
12.09\end{array}$ & $\begin{array}{l}62.66 \pm \\
60.71\end{array}$ & 36 & $\begin{array}{l}101.44 \pm \\
13.55\end{array}$ & $\begin{array}{l}62.88 \pm \\
9.37\end{array}$ \\
\hline Rarely & 163 & $\begin{array}{l}99.90 \pm \\
15.07\end{array}$ & $\begin{array}{l}62.48 \pm \\
1018\end{array}$ & 122 & $99.30 \pm 10$ & $\begin{array}{l}61.55 \pm \\
8.03\end{array}$ \\
\hline \multicolumn{7}{|l|}{ Meals/snacks } \\
\hline$\leq 2$ meals/ day & 4 & $105 \pm 17.32$ & $65 \pm 17.32$ & 8 & $105 \pm 9.25$ & $65 \pm 9.25$ \\
\hline 3-4 meals/ day & 139 & $\begin{array}{l}100.39 \pm \\
14.07\end{array}$ & $\begin{array}{l}62.19 \pm \\
9.70\end{array}$ & 89 & $\begin{array}{l}99.74 \pm \\
10.83\end{array}$ & $\begin{array}{l}61.11 \pm \\
8.97\end{array}$ \\
\hline$\geq 5$ meals/ day & 103 & $\begin{array}{l}99.66 \pm \\
14.50\end{array}$ & $\begin{array}{l}62.66 \pm \\
9.87\end{array}$ & 82 & $100 \pm 11.16$ & $\begin{array}{l}62.98 \pm \\
8.49\end{array}$ \\
\hline
\end{tabular}




\begin{tabular}{|c|c|c|c|c|c|c|}
\hline \multicolumn{2}{|l|}{$\begin{array}{l}\text { Lifestyle (food intake and physical } \\
\text { activity) }\end{array}$} & \multicolumn{3}{|l|}{$\begin{array}{l}\text { Boys } \\
(n=254)\end{array}$} & \multicolumn{2}{|l|}{$\begin{array}{l}\text { Girls } \\
(n=179)\end{array}$} \\
\hline \multicolumn{7}{|l|}{ Watching TV/ PC } \\
\hline$>2$ hours/day & 101 & $\begin{array}{l}102.02 \pm \\
13.08\end{array}$ & $\begin{array}{l}62.97 \pm \\
9.62\end{array}$ & 70 & $\begin{array}{l}101.25 \pm \\
11.53\end{array}$ & $\begin{array}{l}63.57 \pm \\
9.33\end{array}$ \\
\hline 2 hours/ day & 54 & $\begin{array}{l}97.03 \pm \\
12.42\end{array}$ & $\begin{array}{l}60.37 \pm \\
9.85\end{array}$ & 34 & $\begin{array}{l}100.29 \pm \\
10.51\end{array}$ & $\begin{array}{l}61.32 \pm \\
6.43\end{array}$ \\
\hline$<2$ hours/ day & 91 & $\begin{array}{l}99.94 \pm \\
16.31\end{array}$ & $\begin{array}{l}63.07 \pm \\
10.10\end{array}$ & 75 & $\begin{array}{l}99.13 \pm \\
10.57\end{array}$ & $\begin{array}{l}61.18 \pm \\
9.11\end{array}$ \\
\hline \multicolumn{7}{|l|}{ Physical activity } \\
\hline Without targeted physical activity & 31 & $\begin{array}{l}93.06 \pm \\
13.27\end{array}$ & $\begin{array}{l}57.58 \pm \\
10.15\end{array}$ & 41 & $\begin{array}{l}101.70 \pm \\
9.19\end{array}$ & $\begin{array}{l}63.29 \pm \\
9.32\end{array}$ \\
\hline$<420$ minutes/ week & 105 & $\begin{array}{l}102.38 \pm \\
15.56\end{array}$ & $\begin{array}{l}64.38 \pm \\
11.41\end{array}$ & 77 & $\begin{array}{l}101.58 \pm \\
12.41\end{array}$ & $\begin{array}{l}62.85 \pm \\
8.71\end{array}$ \\
\hline$\geq 420$ minutes/week & 110 & $\begin{array}{l}100.04 \pm \\
12.54\end{array}$ & $62 \pm 7.48$ & 61 & $\begin{array}{l}97.13 \pm \\
9.41\end{array}$ & $\begin{array}{l}60.47 \pm \\
7.40\end{array}$ \\
\hline
\end{tabular}

Table 2

Univariate regression coefficients between systolic and diastolic blood pressure with high blood pressure predictors 


\begin{tabular}{|c|c|c|c|c|c|c|c|c|}
\hline \multicolumn{4}{|c|}{ DBP(mmHg) } & \multicolumn{4}{|c|}{ SBP(mmHg) } & \multirow{2}{*}{$\begin{array}{l}\text { Predictor } \\
\text { variables }\end{array}$} \\
\hline R2 & $\begin{array}{l}\text { [95\% Conf. } \\
\text { Interval] }\end{array}$ & $\mathrm{p}$ & Coefficient & $\mathrm{R} 2$ & $\begin{array}{l}\text { [95\% Conf. } \\
\text { Interval] }\end{array}$ & $\mathrm{p}$ & Coefficient & \\
\hline & & & & & & & Referent & $\begin{array}{l}* * \text { BMI } \\
\text { quartile } 1\end{array}$ \\
\hline \multirow[t]{4}{*}{0.2040} & $-0.78,3.78$ & 0.197 & 1.50 & 0.2080 & $0.79,7.06$ & 0.014 & 3.93 & $\begin{array}{l}\text { BMI quartile } \\
2\end{array}$ \\
\hline & $4.79,9.35$ & $>0.001$ & 7.07 & & $6.81,13.08$ & $>0.001$ & 9.50 & $\begin{array}{l}\text { BMI } \\
\text { quartile3 }\end{array}$ \\
\hline & $8.28,12.83$ & $>0.001$ & 10.55 & & $12.39,18.64$ & $>0.001$ & 15.51 & $\begin{array}{l}\text { BMI quartile } \\
4\end{array}$ \\
\hline & & & & & & & Referent & Overweight \\
\hline 0.0005 & $-1.70,2.78$ & 0.636 & 0.54 & 0.0015 & $-1.84,4.32$ & 0.430 & 1.24 & Obesity \\
\hline \multirow[t]{2}{*}{0.0003} & $-2.27,3.35$ & 0.706 & 0.54 & 0.0080 & $-0.24,7.46$ & 0.066 & 3.61 & $\begin{array}{l}\text { Nutrition } \\
\text { screening } \\
\text { pattern }\end{array}$ \\
\hline & & & & & & & Referent & $\begin{array}{l}\text { Fruit } \\
\text { consumption } \\
\text { rarely or } \\
\text { never / day }\end{array}$ \\
\hline \multirow[t]{3}{*}{0.0153} & $1.24,18.29$ & 0.025 & 9.77 & 0.0058 & $-4.84,19.05$ & 0.225 & 7.28 & $\begin{array}{l}\text { Fruit < 2 } \\
\text { serving / day }\end{array}$ \\
\hline & $2.27,18.86$ & 0.013 & 10.57 & & $-3.03,19.87$ & 0.149 & 8.42 & $\begin{array}{l}\text { Fruit } \geq 2 \\
\text { serving / day }\end{array}$ \\
\hline & & & & & & & Referent & $\begin{array}{l}\text { Vegetable } \\
\text { consumption } \\
\text { rarely or } \\
\text { never / day }\end{array}$ \\
\hline \multirow[t]{3}{*}{0.0000} & $-7.01,6.52$ & 0.943 & -0.24 & 0.0002 & $-9.56,9.03$ & 0.955 & -0.26 & $\begin{array}{l}\text { Vegetable }<3 \\
\text { serving / day }\end{array}$ \\
\hline & $-6.81,6.49$ & 0.962 & -0.16 & & $-9.74,8.54$ & 0.897 & -0.60 & $\begin{array}{l}\text { Vegetable } \geq \\
3 \text { serving / } \\
\text { day }\end{array}$ \\
\hline & & & & & & & Referent & $\begin{array}{l}\text { Dairy } \\
\text { consumption } \\
\text { rarely or } \\
\text { never / day }\end{array}$ \\
\hline \multirow[t]{3}{*}{0.0138} & $-3.51,11.96$ & 0.284 & 4.22 & 0.0128 & $-4.66,16.61$ & 0.227 & 5.97 & $\begin{array}{l}\text { Dairy< } 3 \\
\text { serving / day }\end{array}$ \\
\hline & $-5.86,9.32$ & 0.655 & 1.73 & & $-7.74,13.14$ & 0.611 & 2.70 & $\begin{array}{l}\text { Dairy } \geq 3 \\
\text { serving / day }\end{array}$ \\
\hline & & & & & & & Referent & $\begin{array}{l}\text { Fast foods } \\
\text { /junk foods } \\
\text { consumption }\end{array}$ \\
\hline
\end{tabular}




\begin{tabular}{|c|c|c|c|c|c|c|c|c|}
\hline & & & & & & & & $\begin{array}{l}\text { almost every } \\
\text { day }\end{array}$ \\
\hline \multirow[t]{3}{*}{0.0012} & $-3.63,3.47$ & 0.965 & -0.08 & 0.0033 & $-5.92,3.83$ & 0.673 & -1.04 & $\begin{array}{l}\text { Fast foods } \\
\text { /junk foods } \\
1-2 \text { items/ } \\
\text { week }\end{array}$ \\
\hline & $-3.99,2.49$ & 0.650 & -0.75 & & $-6.69,2.20$ & 0.322 & -2.24 & $\begin{array}{l}\text { Fast foods } \\
\text { /junk foods } \\
\text { rarely }\end{array}$ \\
\hline & & & & & & & Referent & $\begin{array}{l}\leq 2 \text { meals or } \\
\text { snacks/day }\end{array}$ \\
\hline \multirow[t]{2}{*}{0.0053} & $-8.71,2.25$ & 0.248 & -3.22 & 0.0043 & $-12.39,2.67$ & 0.206 & -4.85 & $\begin{array}{l}3-4 \text { meals or } \\
\text { snacks / day }\end{array}$ \\
\hline & $-7.70,3.32$ & 0.436 & -2.18 & & $-12.77,2.39$ & 0.179 & -5.18 & $\begin{array}{l}\geq 5 \text { meals or } \\
\text { snacks / day }\end{array}$ \\
\hline \multirow[t]{2}{*}{0.0003} & $-0.43,0.31$ & 0.740 & -0.06 & 0.0029 & $-0.80,0.24$ & 0.268 & -2.90 & $\begin{array}{l}\text { Nutritional } \\
\text { pattern } \\
\text { rating }\end{array}$ \\
\hline & & & & & & & Referent & $\begin{array}{l}\text { Watching } \\
\star \star \star T V / P C> \\
2 \text { hours/day }\end{array}$ \\
\hline \multirow[t]{3}{*}{0.0095} & $-4.90,-0.05$ & 0.045 & -2.47 & 0.0120 & $6.65,0.004$ & 0.050 & -3.32 & $\begin{array}{l}\text { Watching } \\
\text { TV/ PC } 2 \\
\text { hours/ day }\end{array}$ \\
\hline & $-3.00,0.01$ & 0.333 & -0.99 & & $-4.80,0.72$ & 0.147 & -2.04 & $\begin{array}{l}\text { Watching } \\
\text { TV/ PC < } 2 \\
\text { hours/ day }\end{array}$ \\
\hline & & & & & & & Referent & $\begin{array}{l}\text { does not any } \\
\text { targeted } \\
\text { physical } \\
\text { activity }\end{array}$ \\
\hline \multirow[t]{2}{*}{0.0169} & $0.31,5.43$ & 0.028 & 2.87 & 0.0171 & $0.53,7.75$ & 0.024 & 4.05 & $\begin{array}{l}\text { Physical } \\
\text { activity < } \\
420 \\
\text { minutes/ } \\
\text { week }\end{array}$ \\
\hline & $-1.96,3.20$ & 0.636 & 0.622 & & $-2.53,4.57$ & 0.573 & 1.01 & $\begin{array}{l}\text { Physical } \\
\text { activity } \geq \\
420 \\
\text { minutes/ } \\
\text { week }\end{array}$ \\
\hline \multicolumn{9}{|c|}{$\begin{array}{l}* * \text { Body mass index } \quad * * * \text { Television/personal computer. The BMI was categorized as quartile as follows: } 1 \mathrm{st} ; \\
<22.20,2 \mathrm{nd} 22.20-25.22,3 \mathrm{rd} 25.22-28.79,4 \mathrm{th} \geq 28.79 \mathrm{~kg} / \mathrm{m} 2\end{array}$} \\
\hline
\end{tabular}

We examined all the mentioned predictors in three models with hierarchical analysis (Table 3 ) and it has been shown that, in the first model containing gender, age, BMI and being overweight or obese, SBP and DBP had significant correlations with age and BMI directly, and this model could predict $28.1 \%$ of hypertension by SBP (R2 = $0.281)$ and $27.5 \%$ of hypertension by DBP $(R 2=0.275)$. In the second model, we included the consumption of fruit, 
vegetable, dairy products, fast foods /junk foods, meals or snacks, and, nutritional screening score in addition to the variables included in the first model. The results showed that SBP and DBP had significant associations with age and BMI. Moreover, DBP had direct correlation with fruit's consumption. This model could predict $30.7 \%$ of hypertension of SBP $(R 2=0.307)$ and $30.3 \%$ of hypertension of DBP $(R 2=0.303)$ in this age groups. In the third model, we included the watching TV/ PC and PA in addition to the variables included in the second model. The results of the final model showed that SBP had significant positive correlation with age $(p<0.001, B=0.344)$ and $\operatorname{BMI}(p=0.005, B=0.212)$. DBP had also significant correlations with age $(p<0.001, B=0.327), B M I(p=0.007, B=$ 0.207 ), fruit consumption $<2$ serving per day versus never consumption ( $p=0.014, B=0.444)$, fruit $\geq 2$ serving versus never consumption ( $p=0.014, B=0.480)$, and vegetable consumption $<3$ serving versus never consumption $(p=0.045, B=-0.374)$, vegetable consumption $\geq 3$ serving versus never consumption $(p=0.038, B=0.439)$ respectively. DBP had also significant correlations with fast foods /junk foods consumption of 1-2 items per week versus almost every day consumption $(p=0.047, B=-0.177)$. This final model could predict $32.1 \%$ of hypertension $(\mathrm{R} 2=0.321)$.

\section{Table 3}

Blood pressure predictors of obesity and overweight children and adolescents according to lifestyle 


\begin{tabular}{|c|c|c|c|c|c|c|c|}
\hline \multirow[t]{2}{*}{ Model } & \multirow[t]{2}{*}{ Predictor variable } & \multicolumn{3}{|c|}{ SBP (mmHg) } & \multicolumn{3}{|c|}{$\mathrm{DBP}(\mathrm{mmHg})$} \\
\hline & & p & B & $\mathbf{R}^{2}$ & p & B & $\mathrm{R}^{2}$ \\
\hline \multirow[t]{5}{*}{1} & Sex & 0.754 & -0.130 & \multirow[t]{5}{*}{0.281} & 0.537 & -0.025 & \multirow[t]{5}{*}{0.275} \\
\hline & Age & $<0.001$ & 0.354 & & $<0.001$ & 0.356 & \\
\hline & BMI & 0.005 & 0.213 & & 0.007 & 0.205 & \\
\hline & Overweight & \multicolumn{2}{|l|}{ Referent } & & & & \\
\hline & Obesity & 0.747 & -0.016 & & 0.547 & -0.031 & \\
\hline \multirow[t]{21}{*}{2} & Sex & 0.538 & -0.026 & \multirow[t]{21}{*}{0.307} & 0.286 & -0.045 & \multirow[t]{21}{*}{0.303} \\
\hline & Age & $<0.001$ & 0.356 & & $<0.001$ & 0.342 & \\
\hline & BMI & 0.005 & 0.215 & & 0.007 & 0.208 & \\
\hline & Overweight & \multicolumn{2}{|l|}{ Referent } & & & & \\
\hline & Obesity & 0.761 & -0.015 & & 0.545 & -0.013 & \\
\hline & Fruit consumption rarely or never / day & \multicolumn{2}{|l|}{ Referent } & & & & \\
\hline & Fruit $<2$ serving / day & 0.142 & 0.259 & & 0.009 & 0.465 & \\
\hline & Fruit $\geq 2$ serving / day & 0.059 & 0.334 & & 0.003 & 0.533 & \\
\hline & $\begin{array}{l}\text { Vegetable consumption rarely or never / } \\
\text { day }\end{array}$ & \multicolumn{2}{|l|}{ Referent } & & & & \\
\hline & Vegetable $<3$ serving / day & 0.404 & -0.147 & & 0.159 & -2.250 & \\
\hline & Vegetable $\geq 3$ serving / day & 0.345 & -0.168 & & 0.149 & -0.258 & \\
\hline & Dairy consumption rarely or never / day & \multicolumn{2}{|l|}{ Referent } & & & & \\
\hline & Dairy $<3$ serving / day & 0.549 & 0.145 & & 0.463 & 0.144 & \\
\hline & Dairy $\geq 3$ serving / day & 0.692 & 0.079 & & 0.828 & 0.043 & \\
\hline & $\begin{array}{l}\text { Fast foods /junk foods consumption } \\
\text { almost every day }\end{array}$ & \multicolumn{2}{|l|}{ Referent } & & & & \\
\hline & Fast foods /junk foods $1-2$ items/ week & 0.085 & -0.135 & & 0.090 & -0.134 & \\
\hline & Fast foods /junk foods rarely & 0.055 & -0.174 & & 0.081 & -0.159 & \\
\hline & $\leq 2$ meals or snacks/day & \multicolumn{2}{|l|}{ Referent } & & & & \\
\hline & 3-4 meals/ day & 0.659 & -0.058 & & 0.597 & -0.070 & \\
\hline & $\geq 5$ meals/ day & 0.306 & -0.144 & & 0.424 & -0.089 & \\
\hline & Nutritional pattern rating & 0.796 & 0.022 & & 0.656 & 0.038 & \\
\hline \multirow[t]{3}{*}{3} & Sex & 0.537 & -0.026 & \multirow[t]{3}{*}{0.321} & 0.248 & -0.049 & \multirow[t]{3}{*}{0.321} \\
\hline & Age & $<0.001$ & 0.344 & & $<0.001$ & 0.327 & \\
\hline & BMI & 0.005 & 0.2121 & & 0.007 & 0.207 & \\
\hline
\end{tabular}




\begin{tabular}{|c|c|c|c|c|}
\hline \multirow{2}{*}{$\begin{array}{l}\text { Overweight } \\
\text { Obesity }\end{array}$} & \multicolumn{2}{|c|}{ Referent } & \multirow[b]{2}{*}{0.459} & \multirow[b]{2}{*}{$-0.03 c$} \\
\hline & 0.723 & -0.018 & & \\
\hline \multicolumn{3}{|l|}{ Fruit consumption rarely or never / day } & & \\
\hline Fruit $<2$ serving / day & 0.154 & 0.256 & 0.014 & 0.444 \\
\hline Fruit $\geq 2$ serving / day & 0.096 & -0.324 & 0.014 & 0.480 \\
\hline $\begin{array}{l}\text { Vegetable consumption rarely or never / } \\
\text { day }\end{array}$ & \multicolumn{2}{|c|}{ Referent } & & \\
\hline Vegetable $<3$ serving / day & 0.217 & -0.229 & 0.045 & -0.374 \\
\hline Vegetable $\geq 3$ serving / day & 0.189 & -0.277 & 0.038 & -0.439 \\
\hline Dairy consumption rarely or never / day & \multicolumn{2}{|c|}{ Referent } & & \\
\hline Dairy< 3 serving / day & 0.432 & 0.169 & 0.525 & 0.130 \\
\hline Dairy $\geq 3$ serving / day & 0.719 & 0.081 & 0.972 & -0.007 \\
\hline $\begin{array}{l}\text { Fast foods /junk foods consumption } \\
\text { almost every day }\end{array}$ & \multicolumn{2}{|c|}{ Referent } & & \\
\hline Fast foods /junk foods 1-2 items/ week & 0.080 & -0.156 & 0.047 & -0.177 \\
\hline Fast foods /junk foods rarely & 0.118 & -0.212 & 0.060 & -0.255 \\
\hline$\leq 2$ meals or snacks/day & \multicolumn{2}{|c|}{ Referent } & & \\
\hline 3-4 meals/ day & 0.423 & -0.118 & 0.262 & -0.165 \\
\hline$\geq 5$ meals/ day & 0.237 & -0.217 & 0.204 & -0.233 \\
\hline Nutritional pattern rating & 0.724 & 0.103 & 0.344 & 0.277 \\
\hline Watching TV/ PC > 2 hours/day & \multicolumn{2}{|c|}{ Referent } & & \\
\hline Watching TV/ PC 2 hours/ day & 0.169 & -0.093 & 0.059 & -0.128 \\
\hline Watching TV/ PC $<2$ hours/ day & 0.786 & 0.034 & 0.479 & -0.089 \\
\hline does not any targeted physical activity & \multicolumn{2}{|c|}{ Referent } & & \\
\hline Physical activity < 420 minutes/ week & 0.169 & 0.116 & 0.329 & 0.081 \\
\hline Physical activity $\geq 420$ minutes/ week & 0.880 & 0.020 & 0.622 & -0.066 \\
\hline
\end{tabular}

BMI, body mass index; TV/ PC, television/personal computer; the test of Hierarchical regression was performed.

\section{Discussion}

Pediatric hypertension has undergone shift from secondary hypertension to essential hypertension (as the main cause of hypertension in childhood and adolescence) [12]. In the present study, age and BMI were the main predictors of SBP and age, BMI, higher intakes of fruit and fast foods were in positive association and higher intake of vegetables was in negative association with DBP. The univariate analysis showed a significant correlation among adiposity indicators and sedentary lifestyle with SBP and DBP, whereas, higher intake of fruit, also, associated with 
DBP. In accordance to our findings, some other studies reported that increased prevalence of hypertension is associated with higher body mass index $[13,14]$. Higher concentrations of circulating inflammatory cytokines have also been shown to be associated with the atherosclerotic process, and CRP is one of the most susceptible indicators in obese Japanese children $[15,16,17]$. Some studies, also, have shown that increasing age and puberty, especially in adolescent girls, can cause $\operatorname{HBP}[14,18,19]$.

Barba et al reported that in the period close to the completion of puberty, the association between age and BP becomes more evident among girls [18]. In the study of Oliveros et al, the prevalence of prehypertension and stage 2 of hypertension was higher among younger compared with older children, while stage one of hypertension was more prevalent among older children [20]. Among the dietary factors, a positive correlation between hypertension and increased consumption of fruit groups and fast food /junk food consumption, and inverse correlation between hypertension and vegetable groups was observed. Increased fast food consumption containing high amounts of salt, sugar, and fat, is associated with increased obesity [21, 22]. Studies showed that high levels of fats, sugars, and salt intake of fast foods are one of the other possible reasons of increased BP in children and adolescents [23, 24]. Stamler et al. showed that in addition to sodium, several other nutrients including calcium, magnesium, potassium, and fiber are also involved in the pathogenesis of hypertension [25]. One meta-analysis showed that sodium restriction could be beneficial among elderly individuals with hypertension, however, its beneficial effects are low among people with normal BP [26].

The higher sodium and energy contents of fast and junk foods are possible underlying reason of the association between fast food consumption and hypertension. Numerous studies have shown that higher intakes of fruits, vegetables and dairy products could have an effective role in prevention of childhood hypertension due to several nutrients including potassium, magnesium, calcium and fiber $[27,28]$. The positive association between fruits intake and hypertension in our study in contrast with several previous studies, could be attributed to the difference in study design, target group characteristics such as age or gender distribution [27], or taken fruit and vegetables as a one group with no separation of them [28]; while we analyzed fruits and vegetables separately in two independent groups.

Consumption of $100 \%$ fruit juice may be associated with high blood pressure, possibly due to high fructose as well as increased energy consumption, weight gain, and increased uric acid production [29]. Also, consuming whole fruits in high amounts can increase weight due to increased energy intake. Therefore, it could be suggested to study the effects of whole fruits and fruit juices separately in further researches. No significant correlation was found between BP and dairy products in the current study. Greater intakes of dairy products were associated with lower SBP in white but not black children and teens in Dellavalle et al. study, suggesting that greater dairy products intake alone might be not beneficial for all races [30]. There was no significant relationship between meals and hypertension. Some studies showed that the prevalence of obesity declined by increased number of meals [31]. Donin et al. showed that more snacks and meal consumption leads to obesity and cardiovascular problems in children [32]. Also, Toschke et al demonstrated that the prevalence of obesity declines by increasing the number of meals [31]. In the final regression model, we did not observe any significant correlation between BP and PA or watching TV/PC. Torrance et al demonstrated that 40 minutes of moderate to vigorous aerobic-based PA 3-5 days/week is required to reduce BP in obese children [33]. TV commercials, also, influence the food choices of children and encourage people to buy the advertised products [34]. A program comprising screening, early detection and health promotion through school health programs may help to prevent future complications of hypertension [35]. Although in the current study we observed a correlation between BP and television viewing and PA in the 
univariate regression, however these associations had been vanished by including several confounders into the model.

\section{Limitations}

Several factors are possible limitations of the current reports; the family history of hypertension, the effects of other food items including the amount of salt and fat intake and classification of adolescence maturity status, had not been considered.

\section{Abbreviations}

BP: blood pressure; HTN: hypertension; BMI: body mass index; SBP: systolic blood pressure; DBP: diastolic blood pressure; C-IMT: carotid artery intima-media thickness; TV/PC: television/PA

\section{Declarations}

\section{Acknowledgment}

wish to thank the participants in this trial. The authors have no conflicts of interest to report

\section{Authors' contributions}

SHT conducted the analysis and wrote the first draft of the paper, and was coinvestigator responsible for devising on methods and study design. MAJ conducted the analysis and wrote the first draft of the paper, and was coinvestigator responsible for devising on methods and study design. MAF conducted the analysis and wrote the first draft of the paper, and was coinvestigator responsible for devising on methods and study design. FP was coinvestigator responsible for in preparing samples for data preparation and the first draft of the paper. All authors contributed with the interpretation of the results and read and approved the final manuscript.

\section{Funding}

This research has been performed by a grant from Tabriz University of Medical Sciences. The study design was supported by funding source. The funding source had no role in the data collection, analysis, data interpretation, manuscript writing, and the decision on paper submission.

\section{Availability of data and materials}

The related data and material will be available upon request to the corresponding author.

\section{Ethics approval and consent to participate}

This study was approved by IR.TBZMED.REC.1397.692 code by the Ethics Committee of Tabriz University of Medical Sciences. Written informed consent was obtained from participants and parents. 


\section{Consent for publication}

Not applicable.

\section{Competing interests}

The authors declare that they have no competing interests.

\section{References}

1. Juonala M, Magnussen CG, Berenson GS, Venn A, Burns TL, Sabin MA, et al. Childhood adiposity, adult adiposity, and cardiovascular risk factors. N Engl J Med. 2011;365(20):1876-85.

2. Salvadori M, Sontrop JM, Garg AX, Truong J, Suri RS, Mahmud FH, et al. Elevated blood pressure in relation to overweight and obesity among children in a rural Canadian community. Pediatrics. 2008;122(4):e821-e7.

3. Akbari M, Moosazadeh M, Ghahramani S, Tabrizi R, Kolahdooz F, Asemi Z, et al. High prevalence of hypertension among Iranian children and adolescents: a systematic review and meta-analysis. J Hypertens. 2017;35(6):1155-63.

4. Zarei N, Ahmadi A. Nutrition Transition: An Intergenerational Comparison of Dietary Habits among Women of Shiraz. Iran J Public Health. 2015;44(2):269.

5. Garmendia M, Corvalan C, Uauy R. Addressing malnutrition while avoiding obesity: minding the balance. Eur J Clin Nutr. 2013;67(5):513.

6. Malik VS, Willett WC, Hu FB. Global obesity: trends, risk factors and policy implications. Nat Rev Endocrinol. 2013;9(1):13.

7. Menghetti E, Strisciuglio P, Spagnolo A, Carletti M, Paciotti G, Muzzi G, et al. Hypertension and obesity in Italian school children: The role of diet, lifestyle and family history. Nutr Metab Cardiovasc Dis. 2015;25(6):602-7.

8. Moore LL, Bradlee ML, Singer MR, Qureshi MM, Buendia JR, Daniels SR. Dietary Approaches to Stop Hypertension (DASH) eating pattern and risk of elevated blood pressure in adolescent girls. Br J Nutr. 2012;108(9):1678-85.

9. Monzavi R, Dreimane D, Geffner ME, Braun S, Conrad B, Klier M, et al. Improvement in risk factors for metabolic syndrome and insulin resistance in overweight youth who are treated with lifestyle intervention. Pediatrics. 2006;117(6):e1111-e8.

10. Falkner B, Daniels SR. Summary of the fourth report on the diagnosis, evaluation, and treatment of high blood pressure in children and adolescents. Hypertension. 2004;44(4):387-8.

11. Esmailzadeh H, Rajabi F, Rostamigooran N, Majdzadeh R. Iran health system reform plan methodology. Iran J Public Health. 2013;42(Supple1):13.

12. Sorof J, Daniels S. Obesity hypertension in children: a problem of epidemic proportions. Hypertension. 2002;40(4):441-7.

13. Dong Y, Ma J, Song Y, Ma Y, Dong B, Zou Z, et al. Secular trends in blood pressure and overweight and obesity in Chinese boys and girls aged 7 to 17 years from 1995 to 2014. Hypertension. 2018;72(2):298-305.

14. Zeberio N, Malpeli A, Apezteguía M, Carballo MA, González HF. Nutritional status of school-aged children and its relation to blood pressure. Arch Argent Pediatr. 2013;111(2):92-7. 
15. Ogawa Y, Kikuchi T, Nagasaki K, Hiura M, Tanaka Y, Uchiyama M. Usefulness of serum adiponectin level as a diagnostic marker of metabolic syndrome in obese Japanese children. Hypertens Res. 2005;28(1):51.

16. Sorof JM, Alexandrov AV, Garami Z, Turner JL, Grafe RE, Lai D, et al. Carotid ultrasonography for detection of vascular abnormalities in hypertensive children. Pediatr Nephrol. 2003;18(10):1020-4.

17. Järvisalo MJ, Harmoinen A, Hakanen M, Paakkunainen U, Viikari J, Hartiala J, et al. Elevated serum C-reactive protein levels and early arterial changes in healthy children. Arterioscler Thromb Vasc Biol. 2002;22(8):1323-8.

18. Barba G, Casullo C, Dello Russo M, Russo P, Nappo A, Lauria F, et al. Gender-related differences in the relationships between blood pressure, age, and body size in prepubertal children. Am J Hypertens. 2008;21(9):1007-10.

19. Kar S, Khandelwal B. Fast foods and physical inactivity are risk factors for obesity and hypertension among adolescent school children in east district of Sikkim, India. J Nat Sci Biol Med. 2015;6(2):356.

20. Oliveros AM, Molinero A, Cervero M, Magro M, Ponte Y, Partearroyo T. Prevalence Of Hypertension And Hypertension Phenotypes By Age And Gender Among Schoolchildren In Spain: The Mepafac Madrid Regional Community Study. J Hypertens. 2018;36:e215.

21. Alviola IV PA, Nayga Jr RM, Thomsen MR, Danforth D, Smartt J. The effect of fast-food restaurants on childhood obesity: a school level analysis. Econ Hum Biol. 2014;12:110-9.

22. Chou S-Y, Rashad I, Grossman M. Fast-food restaurant advertising on television and its influence on childhood obesity. J Law Econ. 2008;51(4):599-618.

23. Nguyen S, Choi HK, Lustig RH, Hsu C-y. Sugar-sweetened beverages, serum uric acid, and blood pressure in adolescents. J Pediatr. 2009;154(6):807-13.

24. Colín-Ramírez E, Castillo-Martínez L, Orea-Tejeda A, Romero ARV, Castañeda AV, Lafuente EA. Waist circumference and fat intake are associated with high blood pressure in Mexican children aged 8 to 10 years. J Am Diet Assoc. 2009;109(6):996-1003.

25. Stamler J, Caggiula A, Grandits G, Kjelsberg M, Cutler J. Relationship to blood pressure of combinations of dietary macronutrients. Findings of the Multiple Risk Factor Intervention Trial (MRFIT). Circulation. 1996;94(10):2417-23.

26. Midgley JP, Matthew AG, Greenwood CMT, Logan AG. Effect of reduced dietary sodium on blood pressure: a meta-analysis of randomized controlled trials. Jama. 1996;275(20):1590-7.

27. Wu L, Sun D, He Y. Fruit and vegetables consumption and incident hypertension: dose-response meta-analysis of prospective cohort studies. J Hum Hypertens. 2016;30(10):573.

28. Moore LL, Singer MR, Bradlee ML, Djoussé L, Proctor MH, Cupples LA, et al. Intake of fruits, vegetables, and dairy products in early childhood and subsequent blood pressure change. Epidemiology. 2005:4-11.

29. Johnson RJ, Nakagawa T, Sanchez-Lozada LG, Shafiu M, Sundaram S, Le M, et al. Sugar, uric acid, and the etiology of diabetes and obesity. Diabetes. 2013;62(10):3307-15.

30. DellaValle DM, Carter J, Jones M, Henshaw MH. What Is the Relationship Between Dairy Intake and Blood Pressure in Black and White Children and Adolescents Enrolled in a Weight Management Program? J Am Heart Assoc. 2017;6(8):e004593.

31. Toschke AM, Küchenhoff H, Koletzko B, von Kries R. Meal Frequency and Childhood Obesity. Obes Res. 2005;13(11):1933.

32. Donin A, Nightingale C, Owen C, Rudnicka A, Cook D, Whincup P. Takeaway meal consumption and risk markers for coronary heart disease, type 2 diabetes and obesity in children aged 9-10 years: a cross-sectional study. 
Arch Dis Child. 2017;103(5):431-6.

33. Torrance B, McGuire KA, Lewanczuk R, McGavock J. Overweight, PA and high blood pressure in children: a review of the literature. Vasc Health Risk Manag. 2007;3(1):139.

34. Vijayapushpam T, Maheshwar M, Rao DR. A Comparative Analysis of Television Food Advertisements Aimed at Adults and Children in India. Int J Innov Res Sci Eng. 2014;2(6):476-83.

35. Borah PK, Devi U, Biswas D, Kalita HC, Sharma M, Mahanta J. Distribution of blood pressure \& correlates of hypertension in school children aged 5-14 years from North east India. Indian J Med Res. 2015;142:293-300.

\section{Supplementary Files}

This is a list of supplementary files associated with this preprint. Click to download.

- SupplementaryMaterial2.docx 